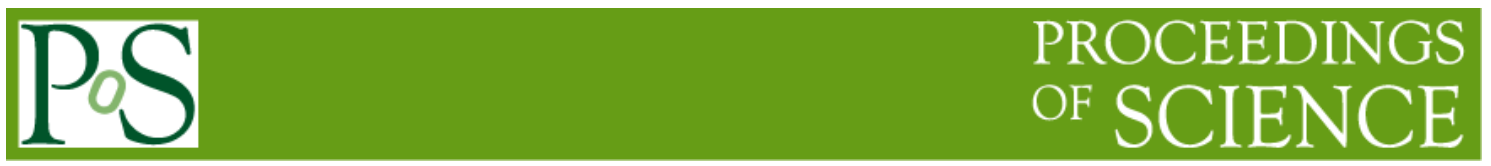

\title{
New results on narrow structure in the pion nucleon elastic scattering from the EPECUR experiment.
}

\section{Anatoly Gridnev}

Petersburg Nuclear Physics Institute

Gatchina, Russia 188300

E-mail: gridnev@pnpi.spb.ru

For EPECUR Collaboration

The new block of high precision data from EPECUR Collaboration is presented. The data has better than $1 \mathrm{MeV}$ resolution in the invariant mass and show some interesting structure for negative pions. The multichannel K-matrix analysis allows explain this structure by two narrow ( 20 MeV) resonances with masses 1686 and $1710 \mathrm{MeV}$. The influence of these resonances on the other channels is discussed also. 
The goal of the EPECUR experiment is search for narrow resonance states by high precision measurements of the pion proton elastic differential cross section. The existence of anti-decuplet of such states was predicted in Ref. [1]. In our experiment, we can study the nucleon like member of it only. The estimated value for mass of this resonance is $1710 \mathrm{MeV}$ as Ref. [1] assumed and $1680 \mathrm{MeV}$ came from modified partial wave analysis [2]. Some structure at this mass region was observed in eta meson photo production off neutron [3, 4]. The data for $\pi$ p elastic scattering (blue points) are shown on Fig. 1 together with results of partial wave analysis WI08 [5] (green lines). The estimated positions of the resonances are shown by arrows. From these pictures, we see that in both places there are some structures but angular dependence is different. There are no such structures in $\pi^{+} p$ scattering (see Fig.2).
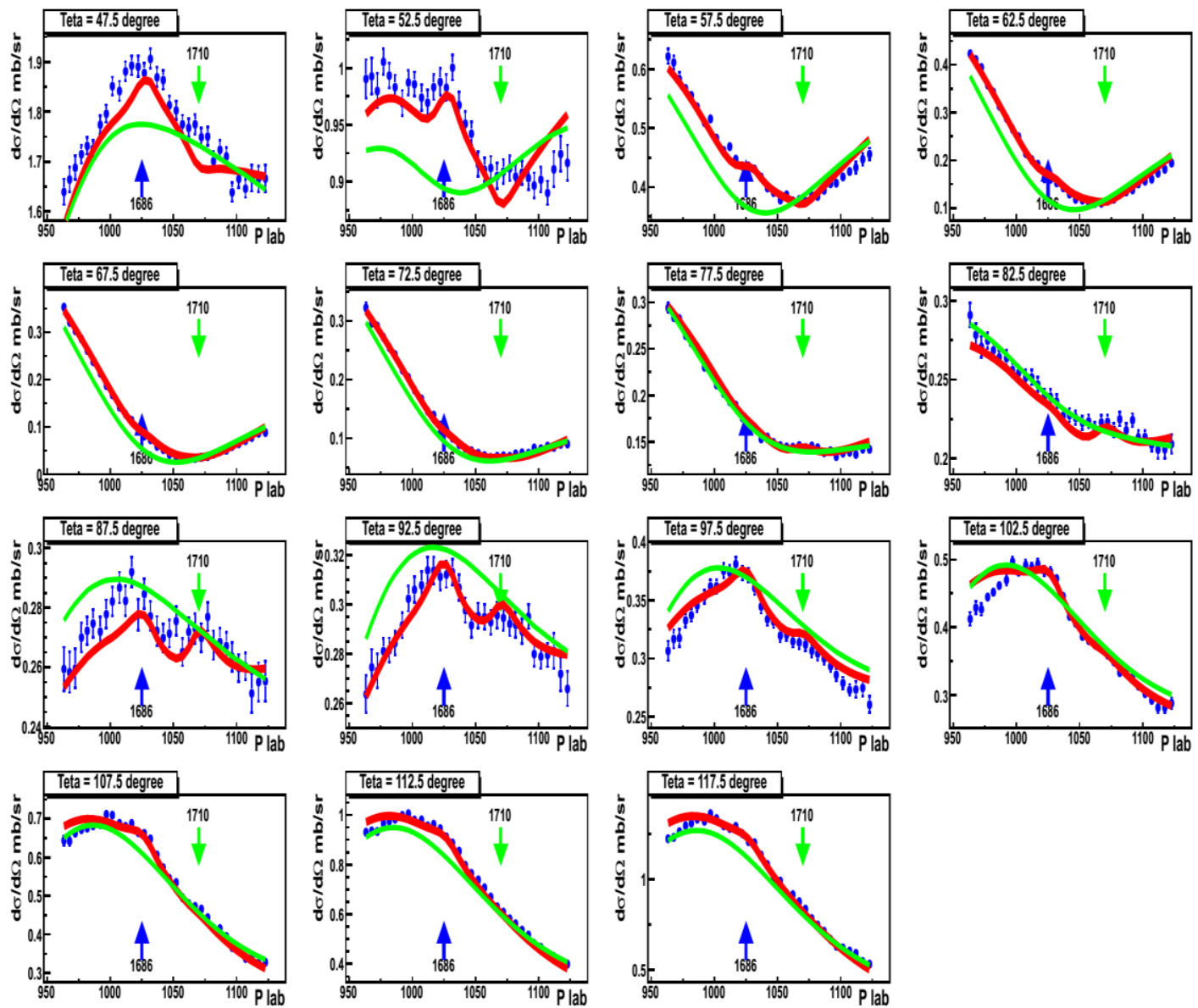

Fig. 1. Blue points are EPECUR $\pi$ p data, green lines show WI08 PWA analysis, red lines are our results.

In the present analysis of our preliminary data, we try to explain these structures by a resonance contribution. To do this, we use the K-matrix approach with effective Lagrangians which were developed in Refs. [6-8] for pion nucleon scattering and then applied for photo production processes [9]. It is assumed that the K-matrix, being a solution of the equation for scattering amplitude, can be considered as a sum of the tree-level Feynman diagrams with the 
effective Lagrangians in the vertices. We include four star PDG resonances in s- and u-channels only with sigma and rho like $t$ channel exchange. To describe the observed structures in $\pi \mathrm{p}$ data two new resonances with isospin $1 / 2$ were added.

The 5-channel analysis is accounted for elastic, $2 \pi$ (effective), $\eta n, K \Lambda$, and $K \Sigma$ channels. Fitted parameters are masses of resonances and coupling constants. The database contained the EPECUR data, total cross-sections for $\pi \mathrm{p} \rightarrow \eta n$ [10] and data on differential cross sections for $\pi \mathrm{p} \rightarrow \mathrm{K} \Lambda$ with $\pi \mathrm{p} \rightarrow \mathrm{K} \Sigma$ [11-14]. To be consistent with data on elastic $\pi \mathrm{N}$ scattering at energies below EPECUR data, we include in the database single energy solutions from WI08 partial wave analysis. The results are shown on Figs. 1 and 2 by red lines.
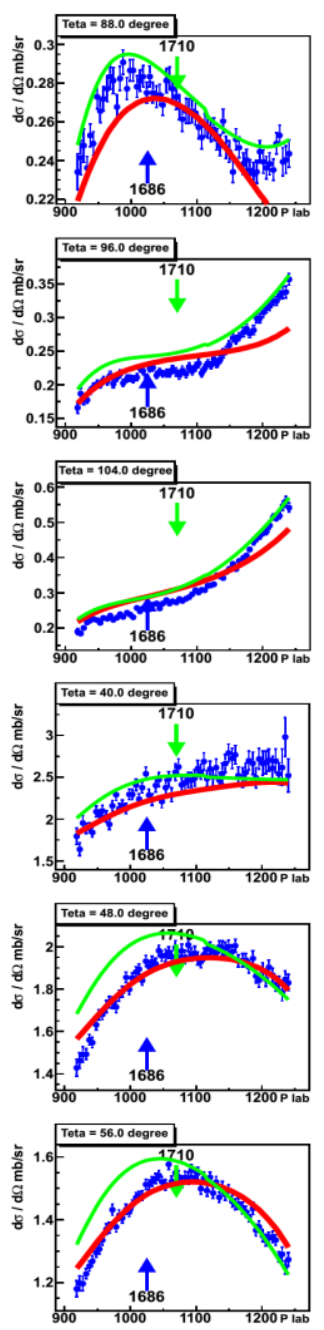
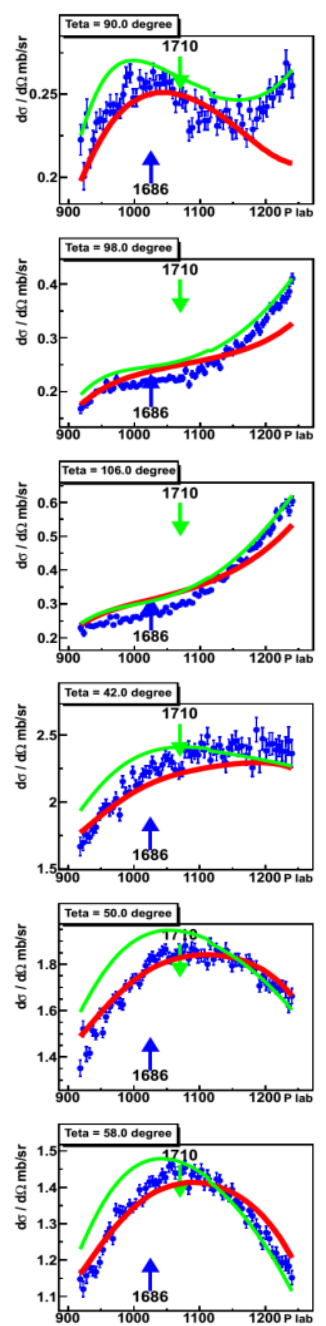
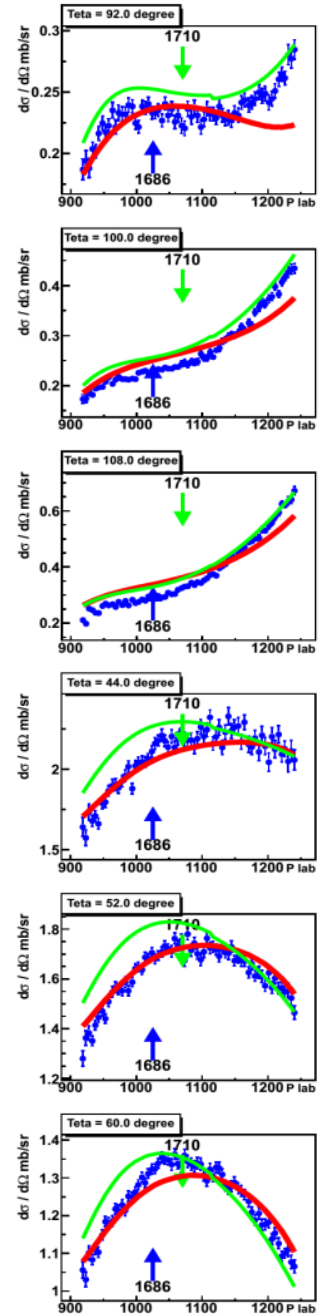
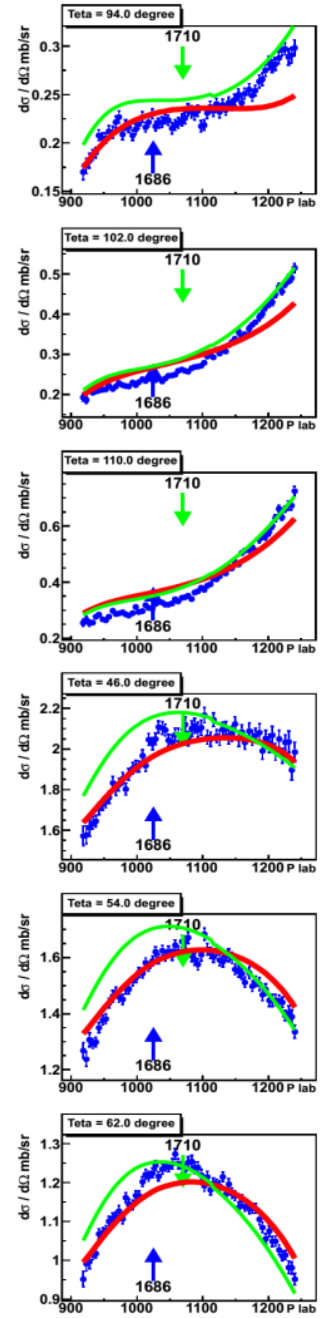

Fig. 2. Blue points show EPECUR $\pi^{+} \mathrm{p}$ data, green lines are WI08 PWA analysis, red lines present our results

It was found that including new resonances decreases $\chi^{2}$ per point for $\pi^{-} \mathrm{p}$ scattering from 6 to $\sim 3.2$ for best fit. We test a different quantum numbers for each resonance and found that the best combination is $S_{11}$ for first resonance and $P_{11}$ for second. However, replacing $S_{11}$ to 
$\mathrm{P}_{11}$ increases $\chi^{2}$ by $15 \%$ only. The parameters of the resonances are shown in the Table1. It should be noted that all of these values are very preliminary being based partial of statistics only.

Table 1. Parameters of the resonances. All quantities are in $\mathrm{MeV}$.

\begin{tabular}{|c|c|c|c|c|c|c|c|}
\hline & $M$ & $\Gamma_{\text {tot }}$ & $\Gamma_{e l}$ & $\Gamma_{2 \pi}$ & $\Gamma_{\eta n}$ & $\Gamma_{K \Lambda}$ & $\Gamma$ \\
& & & & & & & \\
\hline $\mathrm{S}_{11}$ & $1686(1.5)$ & 18.2 & 0.1 & 10. & 8.0 & 0.1 & - \\
\hline $\mathrm{P}_{11}$ & $1710(2.0)$ & 25 & 0.25 & 10. & 5.1 & 0.25 & 9.0 \\
\hline
\end{tabular}

To see how these narrow resonances contribute to other channels, we plot in Fig.3 the total cross-section for the $\pi \mathrm{p} \rightarrow \eta n$ reaction. From this picture, we conclude that the data are not in conflict with our calculations.

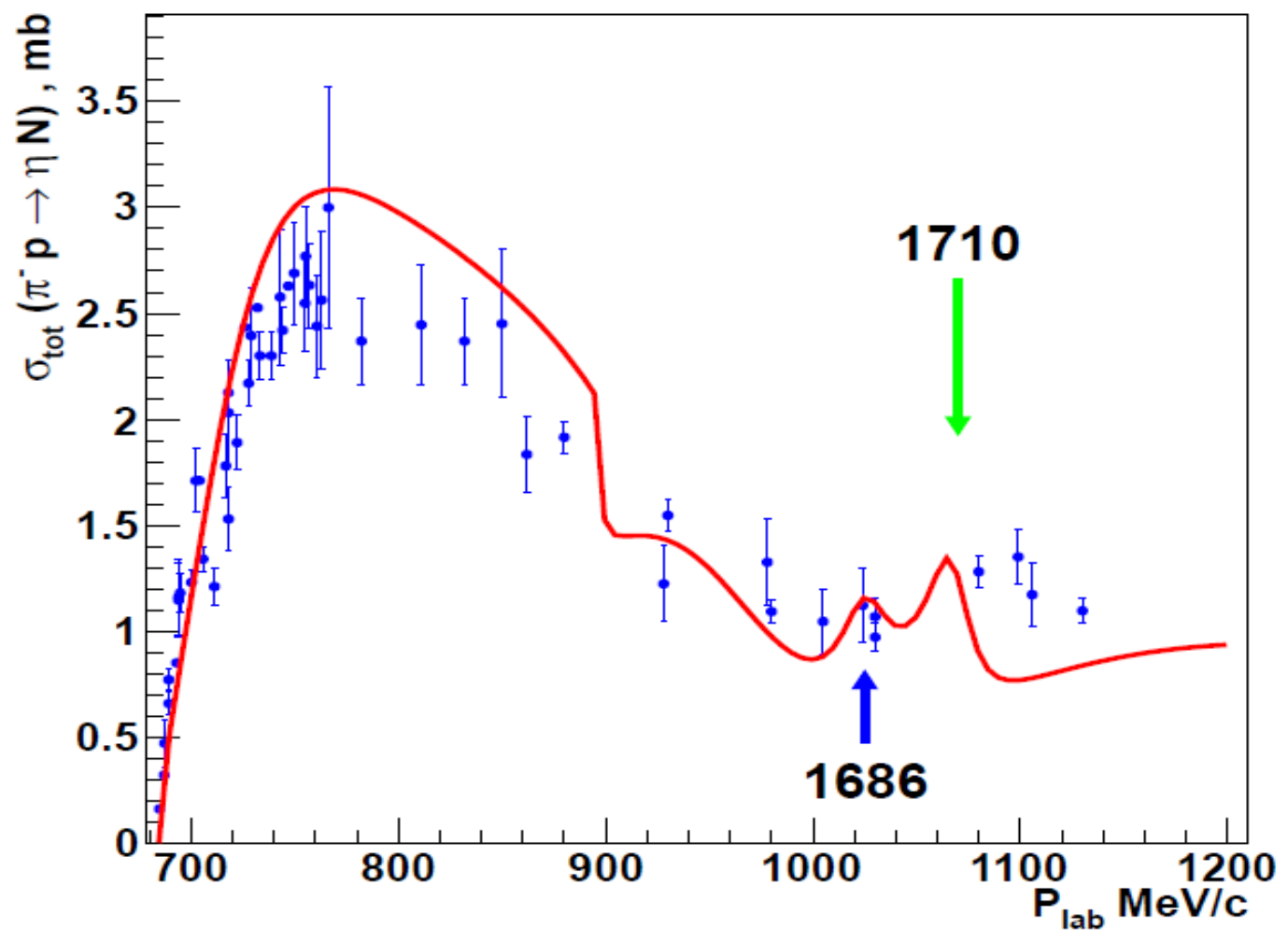

Fig. 3. Total cross-section of the $\pi \mathrm{p} \rightarrow \eta n$ reaction. Data points from Ref. [10]. Red line shows present calculations. 
For observed bump in eta meson photoproduction off the neutron another explanations were suggested based on an interference of well-known resonances $[15,16]$. We not found such a solution in the present paper.

The idea of the cusp effect [17] is under our consideration now. However, in energy region around $1686 \mathrm{MeV}$ possible electromagnetic effects should be taken into account also. Indeed just below $\mathrm{K} \Sigma$ threshold, a bound atomic like state of $\mathrm{K}^{+} \Sigma^{-}$can be created. It is interested that such possibility exists for $\pi \mathrm{p}$ and $\gamma \mathrm{n}$ interactions only. Existence of these electromagnetic effects can be checked by measuring cross-sections for two isospin symmetric reactions $\pi^{-} \mathrm{p} \rightarrow \eta n$ and $\pi^{+} n \rightarrow \eta p$ for example. According isospin symmetry cross-sections of reactions should be the same but $\mathrm{K}^{+} \Sigma^{-}$system exists for the first reaction only.

This work is supported by RFFI grant № 12-02-00981/13.

\section{References}

[1] D. Diakonov et al., Z. Phys. A 359 (1997) 305.

[2] R. A. Arndt et al., Phys. Rev. C 69 (2004) 035208.

[3] V. Kusnetsov et al., Phys. Lett. B 647 (2007) 23.

[4] I. Jagele et al., Phys. Rev. Lett. 100 (2008) 252002.

[5] R. A. Arndt et al., Phys. Rev. C 74 (2006) 045205.

[6] P. F. A.Goudsmit et al., Nucl. Phys. A 575 (1994) 673.

[7] T. Feuster and U. Mozel, Phys. Rev. C 58 (1998) 457.

[8] A. B. Gridnev and N. G. Kozlenko, Eur. Phys. J. A 4 (1999) 187.

[9] T. Feuster and U. Mozel, Phys. Rev. C 59 (1999) 469.

[10] R. A. Arndt et al., Phys. Rev. C 69 (2004) 035213.

[11] R. D. Baker et al., Nucl. Phys. B 145 (1978) 402.

[12] R. D. Baker et al., Nucl. Phys. B 141 (19780) 29.

[13] M. L. Good and R. R. Kofler, Phys. Rev. 183 (1969) 1142.

[14] N. L. Carayannopulos et al., Phys. Rev. 138 (1965) B433.

[15] V. Shklyar et al., Phys. Lett. B 650 (2007) 172.

[16] A. Anisovich et al., Eur. Phys. J. A 41 (2009) 13.

[17] M. Doring and K. Nakayama, Phys. Lett B 683 (2010) 145. 